\title{
Anti-Jamming Schedules for Wireless Data Broadcast Systems
}

\author{
Paolo Codenotti ${ }^{1}$, Alexander Sprintson ${ }^{2}$, and Jehoshua Bruck ${ }^{3}$
}

\begin{abstract}
Modern society is heavily dependent on wireless networks for providing voice and data communications. Wireless data broadcast has recently emerged as an attractive way to disseminate dynamic data to a large number of clients. In data broadcast systems, the server proactively transmits the information on a downlink channel; the clients access the data by listening to the channel. Wireless data broadcast systems can serve a large number of heterogeneous clients, minimizing power consumption as well as protecting the privacy of the clients' locations.

The availability and relatively low cost of antennas resulted in a number of potential threats to the integrity of the wireless infrastructure. In particular, the data broadcast systems are vulnerable to jamming, i.e., the use of active signals to prevent data broadcast. The goal of jammers is to cause disruption, resulting in long waiting times and excessive power consumption.

In this paper we investigate efficient schedules for wireless data broadcast that perform well in the presence of a jammer. We show that the waiting time of client can be reduced by adding redundancy to the schedule and establish upper and lower bounds on the achievable minimum waiting time under different requirements on the staleness of the transmitted data.
\end{abstract}

\section{INTRODUCTION}

Modern society has become heavily dependent on wireless networks to deliver information to diverse users. The emerging wireless infrastructure provides opportunities for building efficient data distribution systems that allow users to access the latest data, such as stock quotes and traffic conditions, at any time, whether they are at home, at their office, or traveling. Wireless data distribution systems also have a broad range of applications in military networks, such as transmitting up-todate battle information to tactical commanders in the field.

Due to their open and ubiquitous nature, wireless information systems are extremely vulnerable to attack and misuse. Wireless systems can be attacked in various ways, depending on the objectives and capabilities of an adversary. Due to high availability and relatively low cost of powerful antennas, jamming, i.e., the use of active signals to prevent data distribution, has emerged as an attractive way of attack. Jamming is a common method of attack in military networks, where the adversary has the capability and the motivation to disrupt communication links.

One common characteristic of wireless infrastructure is an asymmetry between the downlink and uplink channels. Typically, the downlink channel has higher bandwidth and more energy resources than the uplink channel. This intrinsic asymmetry of wireless systems impacts the way information

\footnotetext{
${ }^{1}$ Department of Computer Science, University of Chicago, Chicago, Illinois, USA. Email: paoloc@uchicago.edu

${ }^{2}$ Department of Electrical and Computer Engineering, Texas A\&M University, Texas, USA. Email: spalex@ece.tamu.edu

${ }^{3}$ Parallel and Distributed Computing Group, California Institute of Technology, Pasadena, California, USA. Email: bruck@paradise.caltech.edu
}

is delivered to clients. In particular, the standard client-server paradigm, in which the data transfer is initiated by clients, is not adequate for wireless systems. Wireless data broadcast [1][3] has recently emerged as an attractive way to disseminate data to a large number of clients. In data broadcast systems, the server proactively transmits the information on the downlink channel and the clients access data by listening to the channel. This approach enables the system to serve a large number of heterogeneous clients, minimizing clients' power consumption as well as protecting the privacy of the clients' locations.

Figure 1 depicts a typical data broadcast system. The system includes the following components: the server (scheduler), the broadcast channel, the information source, and the wireless users. The server periodically accesses the information source, retrieves the most recent data, encapsulates it into packets and sends the packets over the broadcast channel. Each transmitted packet carries the most recent update on the state of the information source.

There are two key performance characteristics of a wireless data distribution system. The first characteristic is the waiting time, i.e., the amount of time spent by the client waiting for an update. Waiting time is an important parameter, as timely information delivery is essential for many practical applications. In addition, it is closely related to the amount of power spent by the client to obtain the information. The second characteristic is staleness, i.e., the amount of time that passes from the moment the update is generated, until it is delivered to the client. The staleness of the schedule usually depends on the amount of redundancy used by the system, as information becomes less and less relevant with time.

Jamming Attacks: The goal of the jammer is to disrupt the normal operation of the broadcast system by sending active signals over the channel that interfere with the signal sent by the server (see Figure 1). Jamming signals may result in long waiting time and excessive power consumption of

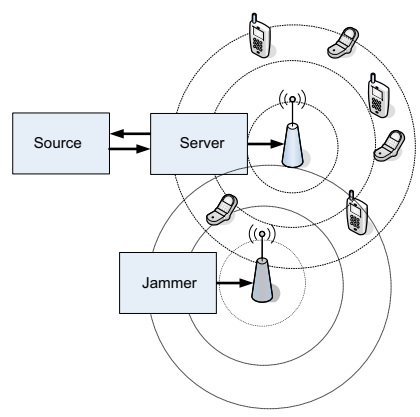

Fig. 1. A typical data broadcast system. 
the clients. The traditional defences against jamming include spread spectrum techniques such as direct sequence and frequency hopping [4], [5]. While these techniques constitute an important tool for combating jamming, an additional protection is required at the packet level due to the following reasons. First, the pseudo-random noise code or frequency hopping sequence may be known to the adversary. Second, even if no information about the spread-spectrum protocol is available to the adversary, it can still destroy a small portion of each transmitted packet by sending a strong jamming signal of short duration. If no other protection mechanism is used at the packet-level, the few destroyed bits will result in dropping of the entire packet.

In this paper we investigate efficient anti-jamming schedules for data broadcast. In our schedules, each update is encoded by an error-correcting code, such as a Reed-Solomon code, which allows the schedule to minimize both waiting time and staleness. As power supply is the most important constraint for practical jammers, we focus on jammers that have certain restrictions on the duration of jamming pulses. To the best of our knowledge, this is the first study that investigates antijamming schedules for wireless data distribution systems.

Related Work: The design of optimal broadcast schedules attracted a large body of research (see [6]-[9] and references therein). Data broadcast over lossy communication channels was studied in [10]. This work proposes efficient coding solutions that reduce performance degradation due to packet loss. Studies [11], [12] focused on the design of universal schedules that guarantee low waiting time for any user, regardless of the access pattern. These studies show that a good universal schedule has to combine both encoding and randomization techniques.

\section{MODEL}

\section{A. Schedules}

As mentioned in the introduction, the goal of the system is to enable each client to access the most updated information is a timely manner. To that end, the server periodically transmits updates, each update captures the most recent state of the information source. We assume that each update includes exactly $k$ information symbols and the transmission of one update without encoding requires one time unit.

Each update is encoded into a packet by using a Maximal Distance Separable (MDS) code, e.g., a Reed-Solomon code [13]. The encoding ensures that any $k$ symbols of the packet are sufficient in order to reconstruct the update. We enumerate updates according to the time of their creation.

Definition 1 (Schedule $\mathcal{S}$ ): A schedule is a sequence $\left\{r_{1}, r_{2}, \ldots\right\}, r_{i} \geq 1$, such that $r_{i} \geq 1$ is the number of time units allocated for the transmission of update $i$.

Note that according to the above definition each packet includes $r_{i} k \geq k$ symbols. A schedule $\mathcal{S}=\left\{r_{1}, r_{2}, \ldots\right\}$ can also be defined by its transmission sequence $\left\{t_{1}, t_{2}, \ldots\right\}$, where $t_{i}$ represents the starting time of the transmission of packet $i$, i.e., $t_{1}=0$ and $t_{i}=\sum_{j=1}^{i-1} r_{j}$ for $i>1$. For example, consider the schedules depicted in Figures 2(a) and 2(b). In the first schedule, each packet is allocated $r$ time units, hence the transmission sequence of the schedule is $0, r, 2 r, \ldots$ The second schedule transmits packets of varying length.

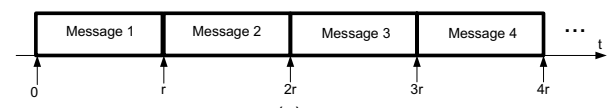

(a)

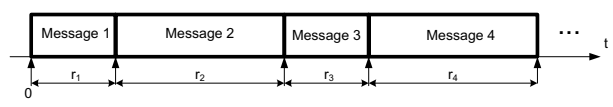

(b)

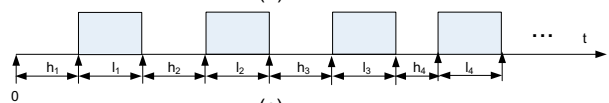

(c)

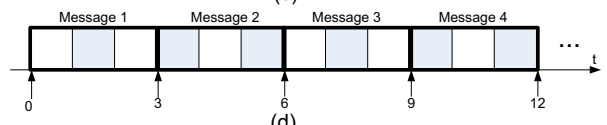

(d)

Fig. 2. Examples of schedules and jamming packets

A wireless client begins to listen to the wireless channel upon a request for new information. In order to satisfy its request, the client must receive at least $k$ symbols from the current or subsequent packets. In particular, if the client fails to receive $k$ symbols from the current packet, it continues to listen to the channel, until it receives at least $k$ symbols from one of the subsequent packets.

The are two key performance characteristics of the schedule: the expected waiting time and the maximum staleness of the received data.

Definition 2 (Waiting time $W T_{t}(\mathcal{S})$ ): Let $\mathcal{S}$ be a broadcast schedule. Suppose that the client's request was placed at time $t$. Let $n$ be the number of the packet currently transmitted over the channel, i.e., the packet for which it holds that $t_{n} \leq$ $t<t_{n+1}$. Let $t^{\prime}$ be the first time the client receives at least $k$ symbols from a packet $n^{\prime}, n^{\prime} \geq n$. Then, the waiting time of the client is defined as $W T_{t}=t^{\prime}-t-1$.

We subtract one from the waiting time, because the client will listen at least that long for any update.

We assume that the clients' requests are distributed uniformly over time. Accordingly, the expected waiting time of the clients is defined as follows:

Definition 3 (Expected Waiting Time $E W T(\mathcal{S})$ ): Let $\mathcal{S}$ be a broadcast schedule. Then, the expected waiting time is defined as follows:

$$
E W T(\mathcal{S})=\lim _{T \rightarrow \infty} \frac{1}{T} \int_{0}^{T} W T_{t}(\mathcal{S}) d t
$$

The waiting time is an extremely important parameter for many time-sensitive applications. In addition, it is closely related to the amount of power spent by the client to obtain the information.

The staleness of the data is defined to be the amount of time that passes from the moment an update is generated until it is delivered to the client. The staleness captures the quality of delivered information, because in dynamic settings the information becomes less and less relevant with time.

Definition $4\left(\right.$ Staleness $S T_{t}(\mathcal{S})$ ): Let $\mathcal{S}$ be a broadcast schedule. Suppose that the client's request was placed at time $t$. Let $n$ be the number of the packet currently transmitted over the channel, i.e., the packet for which it holds that $t_{n} \leq t<t_{n+1}$. Let $t^{\prime}$ be the first time the client receives at least $k$ symbols from an update $n^{\prime}, n^{\prime} \geq n$. Then, the staleness 
of the data is defined to be $S T_{t}=t^{\prime}-t_{n^{\prime}}-1$, where $t_{n^{\prime}}$ is the time the update $n^{\prime}$ was generated.

Again, we subtract one from the staleness, because the client will listen at least that long for any update.

For example, consider the schedule depicted in Figure 2(a). Let $t$ be time the client puts a request for the new information and let $n$ be the number of the packet currently transmitted over the channel. Then, the number of symbols received by the client from packet $n$ is equal to $n_{t}=\left(\left\lceil\frac{t}{r}\right\rceil r-t\right) k$. If $n_{t} \geq k$, then the client will be able to decode this packet, hence its waiting time is zero. Otherwise, the client needs to wait for the next packet, hence its waiting time is equal to $\frac{n_{t}}{k}$. It is easy to verify that if the requests are distributed uniformly over time, then the expected waiting time is $\frac{k}{2 n}=\frac{1}{2 r}$.

While redundant transmission improves the expected waiting time of a schedule, it comes at a price in terms of the staleness of the received data. Indeed, if $n_{t} \geq k$, then the update received by the client at time $t$, was generated in time $\left\lfloor\frac{t}{r}\right\rfloor r$, hence the staleness of the data is $t-\left\lfloor\frac{t}{r}\right\rfloor r$. On the other hand, if $n_{t}<k$, then the client will get a new update, hence the staleness is zero.

A schedule that transmits all updates over time intervals of equal length is referred to as a regular schedule. Regular schedules provide firm guarantees on the staleness of the received data. In particular, schedule $\mathcal{S}_{r}=\{r, r, \ldots\}$ ensures that the staleness of the received information is at most $r-1$ time units. In addition, a regular schedule uses the same encoding for each packet, which simplifies the design of the mobile device and reduces its cost.

\section{Jammer model}

We focus on pulse erasure jammers. Such jammers produce a sequence of pulses, each pulse results in an erasure in the channel.

Definition 5 (Jamming Sequence $\mathcal{J}$ ): A jamming sequence is a sequence $\left\{h_{1}, l_{1}, h_{2}, l_{2}, \ldots\right\}$, such that $h_{1}$ is the beginning time of the first pulse, $l_{i}$ is the length of pulse $i$, and $h_{i}, i \geq 2$ is the length of time interval between pulses $i-1$ and $i$. Figure 2(c) depicts an example of a jamming sequence.

It has been recognized [14] that the power supply is the most important limitation for the majority of practical jammers. A typical jammer is powered by a battery, which can be recharged from an external source, such as a solar cell array. Accordingly, in our model, we limit the length of pulses in the jamming sequence by a constraint $l^{\max }$, i.e., $l_{i} \leq l^{\max }$ for all $i \geq 1$. Since after each pulse the battery must be recharged we also constrain the length of the interval between two consecutive pulses to be at least $h^{\mathrm{min}}$, i.e., $h_{i} \geq h^{\mathrm{min}}$ for all $i \geq 2$. We refer to a jamming sequence that satisfies the energy limitations as an admissible jamming sequence.

We denote by $W T_{t}(\mathcal{S}, \mathcal{J})$ the waiting time of schedule $\mathcal{S}$ in the presence of jammer $\mathcal{J}$. Similarly, the expected waiting time of a schedule $\mathcal{S}$ in the presence of jammer $\mathcal{J}$ is denoted by $\operatorname{EWT}(\mathcal{S}, \mathcal{J})$, i.e., $\operatorname{EWT}(\mathcal{S}, \mathcal{J})=$ $\lim _{T \rightarrow \infty} \frac{1}{T} \int_{0}^{T} W T_{t}(\mathcal{S}, \mathcal{J}) d t$

For example, suppose that a jamming sequence $\mathcal{J}=$ $\{1,1,1, \ldots\}$ is applied to the schedule $\mathcal{S}=\{3,3, \ldots\}$ (see Figure $2(\mathrm{~d})$ ). In this case, the expected waiting time is equal to

\begin{tabular}{||c|c|c||}
\hline \hline$r$ & Lower Bound & Upper Bound \\
\hline$r<2$ & $\infty$ & $\infty$ \\
\hline $2 \leq r<3$ & $1+\frac{2}{r}$ & $1+\frac{2}{r}$ \\
\hline$r=3$ & $\frac{23}{18}$ & $\frac{23}{18}$ \\
\hline $3<r<1+\frac{\sqrt{51}}{3}$ & $\frac{23}{6 r}$ & $\frac{3}{4}+\frac{2 \delta^{2}-\delta+10}{4 r}$ \\
\hline $1+\frac{\sqrt{51}}{3} \leq r<4$ & $\frac{r^{2}-2 r+3}{2 r}$ & $\frac{3}{4}+\frac{2 \delta^{2}-\delta+10}{4 r}$ \\
\hline $2 i \leq r<2 i+1$, & $\frac{3}{4}+\frac{10+r-\lfloor r\rfloor}{4 r}$ & $\frac{3}{4}+\frac{10+r-[r\rfloor}{4 r}$ \\
$i=2,3 \ldots$ & $\frac{3\lfloor r\rfloor+11}{4 r}$ & $\frac{3}{4}+\frac{2 \delta^{2}-\delta+10}{4 r}$ \\
\hline $2 i+1 \leq r<2 i+\sqrt{3}$ & $\frac{3}{4}+\frac{2 \delta^{2}-5 \delta+10}{4 r}$ & $\frac{3}{4}+\frac{2 \delta^{2}-\delta+10}{4 r}$ \\
$i=2,3, \ldots$ & & \\
\hline $2 i+\sqrt{3} \leq r<2(i+1)$ & & \\
\hline
\end{tabular}

TABLE I

UPPER AND LOWER BOUNDS ON THE MAXIMUM EXPECTED WAITING TIME $M W T\left(\mathcal{S}_{r}\right)$ OF REGULAR SCHEDULES $\mathcal{S}_{r}=\{r, r, \ldots\}$. HERE,

$$
\delta=2\left\lceil\frac{r}{2}\right\rceil-r
$$

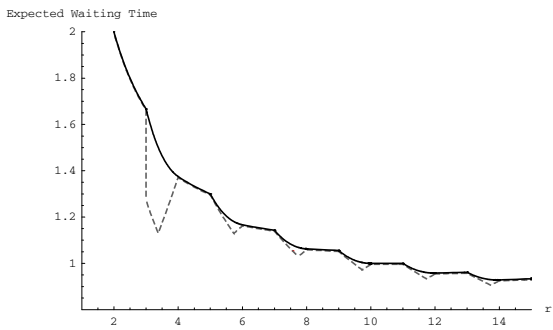

Fig. 3. The lower and upper bounds on $M W T\left(\mathcal{S}_{r}\right)$. The lower and upper bounds are marked by solid and dashed lines, respectively.

$E W T(\mathcal{S}, \mathcal{J})=11 / 12$, which is by $\frac{5}{12}$ more than the expected waiting time of the same schedule without jamming.

For a given schedule $\mathcal{S}$, we define its maximum waiting time $M W T(\mathcal{S})$ to be the maximum value of $\operatorname{EWT}(\mathcal{S}, \mathcal{J})$ over all admissible jamming sequences $\mathcal{J}$. The maximum waiting time characterizes the worst-case behavior of the schedule in the presence of an adversarial jammer.

\section{Results}

In this study we investigated the performance of broadcast schedules in the presence of a jammer. First, we established lower and upper bounds on the maximum expected waiting time $M W T\left(\mathcal{S}_{r}\right)$ of regular schedules $\mathcal{S}_{r}=\{r, r, \ldots\}$. Then, we extended our results for general schedules and investigated the trade-off between the maximum expected waiting time $M W T\left(\mathcal{S}_{r}\right)$ and the staleness constraint.

Our results for regular schedules are summarized in Table I and in Figure 3. All of our lower bounds are constructive as we present jamming sequences that yield the expecting waiting times equal to the value of the lower bound. We also identify optimal jamming sequences for many classes of regular schedules. All results are up to terms of order $\varepsilon=\frac{1}{k}$.

It is important to note that the size of the packet $r$ in a regular schedule $\mathcal{S}_{r}$ is closely related to the staleness of the delivered information. Indeed, the maximum staleness of the data is always lower or equal to $r-1$, while its average staleness does not exceed $\frac{r-1}{r}$. Hence our results establish a 


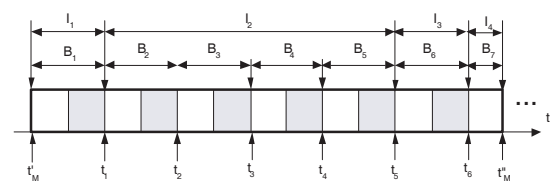

Fig. 4. Division of a interval $I_{M}$ into blocks and subintervals $I_{1}, \ldots, I_{4}$.

trade-off between the expected waiting time of the clients and the staleness. In particular, we identify the best schedule for any given staleness constraint. We observe that the schedule $\mathcal{S}_{3}$ has a clear advantage over other schedules: it achieves low expected waiting time with minimum penalty in terms of the staleness of the delivered data.

In addition, we established upper and lower bounds on the worst case waiting time $M W T(\mathcal{S})$ for a general class of non-regular schedules. This class includes schedules in which the length of each packet is different and the schedules that employ randomization, i.e., the length of each packet is distributed according to some probability distribution. We assume that in the case of random schedules the jammer knows the probability distribution but has no access to the server's random bits.

Theorem 6: Let $\mathcal{S}$ be a schedule and let $r$ be the expected length of the packets in $\mathcal{S}$. Then the worst case expected waiting time $M W T(\mathcal{S})$ of the schedule in the presence of an admissible jammer is bounded by

$$
\frac{3}{4}+\frac{3}{2 r} \leq M W T(\mathcal{S}) \leq \frac{3}{4}+\frac{11}{4 r}
$$

Due to the space constraints, most of the proofs and technical details are omitted from this paper and can be found in [15].

\section{UPPER BOUNDS FOR REGULAR SCHEDULES}

In this section we establish upper bounds on the optimal jamming sequences for regular schedules $\mathcal{S}_{r}$ for a certain range of values of $r$.

We begin by introducing the notion of added waiting time. Let $I=\left[t^{\prime}, t^{\prime \prime}\right]$ be a time interval, $\mathcal{S}$ be a schedule, and $\mathcal{J}$ be a jamming sequence. Then, the added waiting time for interval $I$ is defined to be $A W T(I, \mathcal{J})=\int_{t^{\prime}}^{t^{\prime \prime}}\left(W T_{t}(\mathcal{S}, \mathcal{J})-\right.$ $\left.W T_{t}(\mathcal{S})\right) d t$. Intuitively, $A W T(I, \mathcal{J})$ captures the total amount of additional waiting time experienced by clients' requests that arrive during interval $I$ due to jamming.

Let $\mathcal{S}_{r}, r \geq 3$ be a regular schedule, $\mathcal{J}$ be an admissible jamming sequence, and $I_{M}=\left[t_{M}^{\prime}, t_{M}^{\prime \prime}\right]$ be a time interval allocated for transmission of a single update. Our goal is to obtain an upper bound on $A W T(I, \mathcal{J})$. Such a bound would immediately translate in an upper bound on $\operatorname{MWT}\left(\mathcal{S}_{r}\right)$.

We denote by $t_{1}, \ldots, t_{h}$ the finishing times of the jamming pulses that belong to $I_{M}$ and divide $I_{M}$ into $h+1$ blocks $B_{1}, \ldots, B_{h+1}$, such that $B_{1}=\left[t_{M}^{\prime}, t_{1}\right], B_{i}=\left[t_{i-1}, t_{i}\right]$ for $2 \leq i \leq h$, and $B_{h+1}=\left[t_{h}, t_{M}^{\prime \prime}\right]$ (See Figure 4). We also divide $I_{M}$ into subintervals $I_{1}, \ldots, I_{4}$ such that that $I_{1}=B_{1}$, $I_{2}$ includes blocks $B_{2}, \ldots, B_{h-1}, I_{3}=B_{h}$, and $I_{4}=B_{h+1}$. We denote by $T_{i}$ the length of the subinterval $I_{i}$.

In the following lemma we establish an upper bound for each of the subintervals $I_{1}, \ldots, I_{4}$.

\section{Lemma 7:}

$$
\begin{aligned}
& A W T\left(I_{1}, \mathcal{J}\right) \leq \begin{cases}T_{1}^{2} / 2 & \text { if } T_{1} \leq 1 \\
T_{1}-1 / 2 & \text { if } 1 \leq T_{1} \leq 2\end{cases} \\
& A W T\left(I_{2}, \mathcal{J}\right) \leq \begin{cases}\frac{3 T_{2}+2 \delta^{2}-5 \delta}{4} & \text { if }\left\lfloor T_{2}\right\rfloor \text { is odd }, \\
\frac{3}{4}\left(\left\lfloor T_{2}\right\rfloor\right) & \text { otherwise, }\end{cases} \\
& A W T\left(I_{3}, \mathcal{J}\right) \leq \begin{cases}\frac{2 T_{4}\left(T_{3}-2\right)+T_{3}^{2}+2 T_{3}-1}{2} & \text { if } T_{4}<1 \\
\frac{T_{3}^{2}-1}{T_{4}} & \text { if } T_{4} \geq 1\end{cases} \\
& A W T\left(I_{4}, \mathcal{J}\right) \leq \begin{cases}T_{4} & \text { if } T_{4}<1 \\
\frac{-T_{4}^{2}+4 T_{4}-1}{4} & \text { if } T_{4} \geq 1\end{cases}
\end{aligned}
$$

where $\delta=\left\lceil T_{2}\right\rceil-T_{2}$.

Proof: See [15].

We assume, without loss of generality, that intervals $I_{1}, I_{3}$, or $I_{4}$ do not contain an unjammed interval whose length is longer than one time unit. Indeed, if this is the case, such an interval can be shortened at the expense of one of the unjammed intervals in $I_{2}$, with no increase in the value of $A W T\left(I_{M}, \mathcal{J}\right)$. This implies that $T_{1}, T_{3}, T_{4} \leq 2$. The added waiting time for the interval $I_{M}$ equals to the sum of the added waiting times for its subintervals $I_{1}, \ldots, I_{4}$, i.e., $A W T\left(I_{M}, \mathcal{J}\right)=$ $A W T\left(I_{1}, \mathcal{J}\right)+A W T\left(I_{2}, \mathcal{J}\right)+A W T\left(I_{3}, \mathcal{J}\right)+A W T\left(I_{4}, \mathcal{J}\right)$.

An upper bound on $A W T\left(I_{M}, \mathcal{J}\right)$ can be found by solving the following maximization program:

$$
\begin{array}{lll}
\text { maximize } & A W T\left(I_{M}, \mathcal{J}\right) & \\
\text { subject to } & & \\
& T_{1}+T_{2}+T_{3}+T_{4}=r & \\
& T_{i} \geq 0 & i=1, \ldots, 4 \\
& T_{i} \leq 2 & i=1,2,4
\end{array}
$$

It can be shown, using the tools of the theory of constrained optimization that for $r \geq 3$, the optimal value of $A W T\left(I_{M}, \mathcal{J}\right)$ is achieved when the following conditions are satisfied: (a) $T_{1}=r-T_{2}-T_{3}-T_{4}$, (b) $T_{2}=2\left\lfloor\frac{r-3}{2}\right\rfloor$ (c) $T_{3}=2$ (d) $T_{4}=1$.

If $r \geq 4$ and $\lfloor r\rfloor$ is an even number this implies the following upper bound on $A W T\left(I_{M}, \mathcal{J}\right)$ :

$$
A W T\left(I_{M}, \mathcal{J}\right) \leq \frac{3 r+8+r-\lfloor r\rfloor}{4},
$$

which, in turn, implies that

$$
\operatorname{MWT}\left(\mathcal{S}_{r}\right) \leq \frac{3}{4}+\frac{r-\lfloor r\rfloor+10}{4 r} . .
$$

If $r \geq 3$ and $\lfloor r\rfloor$ is an odd number, these condition imply the following upper bound on $A W T\left(I_{M}, \mathcal{J}\right)$ :

$$
A W T\left(I_{M}, \mathcal{J}\right) \leq \frac{3 r+8-\delta+2 \delta^{2}}{4},
$$

where $\delta=2\left\lceil\frac{r}{2}\right\rceil-r$. This, in turn, implies that

$$
M W T\left(\mathcal{S}_{r}\right) \leq \frac{3}{4}+\frac{2 \delta^{2}-\delta+8}{4 r} .
$$

We conclude our discussion by the following theorem:

Theorem 8: Let $\mathcal{S}_{r}=\{r, r, \cdots\}$ be a regular schedule. Then if $r \geq 3$ and the integer part of $r$ is an odd number 


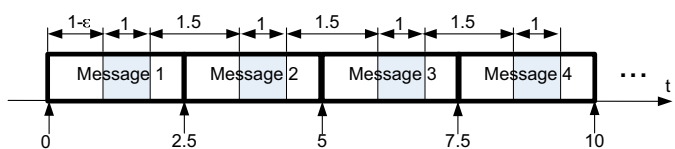

(a)

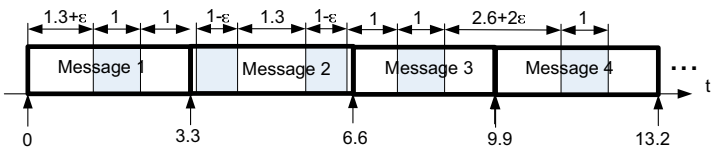

(b)

Fig. 5. Jamming sequences for regular schedules $\mathcal{J}_{r}$ (a) $\mathrm{r}=2.5$ (b) $r=3.3$

then $M W T\left(\mathcal{S}_{r}\right) \leq \frac{3}{4}+\frac{10+2 \delta^{2}-\delta}{4 r}$, where $\delta=2\left\lceil\frac{r}{2}\right\rceil-r$. If $r \geq 4$ and the integer part $\lfloor r\rfloor$ of $r$ is an even number, then $M W T\left(\mathcal{S}_{r}\right) \leq \frac{3}{4}+\frac{r-\lfloor r\rfloor+10}{4 r}$.

\section{LOWER BOUNDS}

Theorem 9: Let $\mathcal{S}_{r}$ be a regular schedule. Then, up to terms of order $\varepsilon=\frac{1}{k}$,

$$
\begin{aligned}
& M W T\left(\mathcal{S}_{r}\right) \geq \\
& \begin{cases}1+\frac{2}{r} & \text { if } 2 \leq r<3 \\
\frac{23}{6 r} & \text { if } 3 \leq r<1+\sqrt{\frac{51}{3}} \\
\frac{r^{2}-2 r+3}{2 r} & \text { if } 1+\sqrt{\frac{51}{3}} \leq r<4 \\
\frac{3}{4 r}+\frac{10+r-\lfloor r\rfloor}{4 r} & \text { if } 2 i \leq r<2 i+1 \\
\frac{3\lfloor r\rfloor+11}{4 r} & i=2,3, \ldots \\
& \text { if } 2 i+1 \leq r<2 i+\sqrt{3} \\
\frac{3}{4}+\frac{2 \delta^{2}-5 \delta+10}{4 r} & i=2,3, \ldots \\
& i f=2,3, \ldots\end{cases}
\end{aligned}
$$

where $\delta=\lceil r\rceil-r$.

Proof: We prove the theorem by presenting, for each schedule $\mathcal{S}_{r}, r \geq 2$ a jamming sequence $\mathcal{J}_{r}$ such that $E W T\left(\mathcal{S}_{r}, \mathcal{J}_{r}\right)$ is equal to the values of the lower bounds stated in the theorem.

For $2 \leq r<3, \mathcal{J}_{r}=\{1-\varepsilon, 1,1+\delta, 1,1+\delta, \ldots\}$, where $\delta=r-3$ and $\varepsilon=\frac{1}{k}$. An example of this schedule for $r=2.5$ is depicted on Figure 5(a). For $3 \leq r<1+\frac{\sqrt{51}}{3}, \mathcal{J}_{r}=$ $\{1+\delta+\varepsilon, 1,1,1-\varepsilon, 1+\delta, 1-\varepsilon, 1,1,2+2 \varepsilon+2 \delta, 1,1,1-\varepsilon, 1+$ $\delta, 1-\varepsilon, 1,1,2+2 \varepsilon+2 \delta, \ldots\}$, where $\delta=r-\lfloor r\rfloor$ and $\varepsilon=\frac{1}{k}$. This schedule is depicted on Figure 5(b). For $1+\frac{\sqrt{51}}{3} \leq r<4$, $\mathcal{J}_{r}=\left\{1-\varepsilon, 1,1, \delta, 1,1,1, \delta, \ldots\right.$, where $\varepsilon=\frac{1}{k}$ and $\delta=r-3$. For $2 i \leq r<2 i+1, i=2,3, \ldots, \mathcal{J}=\{1-\varepsilon, 1,1+$ $2 \varepsilon, 1 \cdots \overline{1}, 1+\delta-2 \varepsilon, 1,1+2 \varepsilon, 1, \cdots, 1,1+\delta-2 \varepsilon, \cdots\}$, where $\varepsilon=\frac{1}{k}$ and $\delta=r-\lfloor r\rfloor$. For $2 i+1 \leq r<2 i+\sqrt{3}, i=2,3, \ldots$, $\mathcal{J}=\{1-\varepsilon, 1,1+\delta+2 \varepsilon, 1,2-2 \varepsilon, 1,1+\delta+2 \varepsilon, \ldots\}$, where $\varepsilon=\frac{1}{k}$ and $\delta=r-\lfloor r\rfloor$. For $2 i+\sqrt{3} \leq r<2(i+1)$, $i=2,3, \ldots, \mathcal{J}_{R}=(1-\varepsilon, 1,1, \delta, 1, \cdots, 1, \delta, \cdots), \mathcal{J}_{R}=$ $\{1-\varepsilon, 1,1, \delta, 1, \cdots, 1, \delta, \cdots\})$, where $\varepsilon=\frac{1}{k}$ and $\delta=r-\lfloor r\rfloor$.

\section{CONCLUSION}

We investigated the design of efficient anti-jamming schedules for wireless data distribution systems. For such schedules, waiting time and staleness are the key performance parameters.
The goal of the jammer is to induce large delays in data transmission and to increase the staleness of the data by forcing the schedule to transmit the data with high level of redundancy.

Our contribution can be summarized as follows. First, we identify optimal and near optimum jamming strategies for the important class of regular schedules. In such schedules, the same encoding is used for all packets, which simplifies the design of the mobile device and reduces its cost. Next, we provided lower and upper bounds on the performance of more general class of non-regular schedules. Our results establish a trade-off between the expected waiting time of the client and the staleness of the information in the presence of a jammer.

As a future research, we intend to extend our results to the case in which the broadcast channel is shared by two or more information sources. We also would like to investigate the performance of random anti-jamming schedules for wireless data broadcast.

\section{REFERENCES}

[1] T. Imielinski, S. Viswanathan, and B.R. Badrinath. Data on Air: Organization and Access. IEEE Transactions on Knowledge and Data Engineering, 9(3):353-372, 1997.

[2] D. Cheriton. Dissemination-Oriented Communication Systems. Technical report, Stanford University, 1992.

[3] S. Acharya, R. Alonso, M. Franklin, and S. Zdonik. Broadcast Disks: Data Management for Asymmetric Communication Environments. In Proceedings of ACM SIGMOD, pages 199-210, San Jose, CA, May 1995.

[4] A. J. Viterbi. Spread Spectrum Communications: Myths and Realities. IEEE Communication Magazine, 50th Anniversary Commemorative issue, pages 34-41, May 2002.

[5] M. K. Simon, J. K. Omura, R. A. Scholtz, and B. K. Levitt. Spread Spectrum Communications Handbook. McGraw Hill, 1995.

[6] K. Foltz and J. Bruck. Time Division is Better than Frequency Division for Periodic Internet Broadcast of Dynamic Data. In Proceedings of International Symposium on Information Theory (ISIT), page 158, Washington, DC, July 2001.

[7] K. Foltz and J. Bruck. Splitting Schedules for Internet Broadcast Communication. IEEE Transactions on Information Theory, 48(2):345358, February 2002.

[8] K. Foltz and J. Bruck. Robustness of Time-Division Schedules for Internet Broadcast. In Proceedings of International Symposium on Information Theory (ISIT), page 383, Lausanns, Switzerland, June-July 2002.

[9] K. Foltz, L. Xu, and J. Bruck. Scheduling for Efficient Data Broadcast over Two Channels. In Proceedings of International Symposium on Information Theory (ISIT), page 113, Chicago, IL, USA, June 2004.

[10] K. Foltz, L. Xu, and J. Bruck. Coding and Scheduling for Efficient LossResilient Data Broadcasting. In Proceedings of International Symposium on Information Theory (ISIT), page 413, Yokohama, Japan, June-July 2003.

[11] M. Langberg, A. Sprintson, and J. Bruck. Optimal Universal Schedules for Discrete Broadcast. In Proceedings of International Symposium on Information Theory (ISIT), Chicago, IL, USA, June-July 2004.

[12] M. Langberg, A. Sprintson, and J. Bruck. Staleness vs. Waiting Time in Universal Discrete Broadcast. In Submitted to ISIT'2005. available from http://www.paradise.caltech.edu/spalex/conferences.html.

[13] W.C. Huffman and V.S. Pless. Handbook of Coding Theory, vol. 1. Elsevier Science, 1998.

[14] G. Lin and G. Noubir. On Link Layer Denial of Service in DATA Wireless LANs. Wiley Journal on Wireless Communications and Mobile Computing, to appear.

[15] P. Codenotti, A. Sprintson, and J. Bruck. Anti-Jamming Schedules for Wireless Broadcast Systems. ETR070, California Institute of Technology, July, 2005. Available from: http://www.paradise.caltech.edu/ETR.html. 